\title{
LA EDUCACIÓN FÍSICA EN LA PRENSA PEDAGÓGICA MEXICANA EN LOS INICIOS DEL SIGLO XX. UN CAMPO EN DISPUTA, LO ESCOLAR Y EL GÉNERO ${ }^{1}$
}

\author{
THE PHYSICAL EDUCATION IN THE MEXICAN PEDAGOGICAL PRESS AT THE \\ EARLY TWENTIETH CENTURY. A FIELD IN DISPUTE, SCHOOL AND GENDER
}

\author{
EDUCAÇÃO FÍSICA NA IMPRENSA PEDAGÓGICA MEXICANA NO INÍCIO \\ DO SÉCULO XX. UM CAMPO DISPUTADO, ESCOLA E GÊNERO
}

Georgina Ramírez Hernández²

\section{Resumen}

\begin{abstract}
Este artículo de reflexión tiene el objetivo de analizar las prácticas culturales de la educación física como parte de una educación corporal desde lo escolar y el género durante la primera década del siglo xx mexicano a partir de las revistas pedagógicas. Ello parte de la consideración, desde la historia de la educación mexicana, que, a principios del siglo Xx, la educación física vivió uno de los periodos más importantes en su historia. Se gestaron una serie de procesos en torno a ella, considerada tanto disciplina escolar como práctica cotidiana, bajo el cobijo de las reformas implementadas por el presidente Porfirio Díaz, en particular en la década de 1900-1910, las cuales generaron disputas en su contra al ubicarla por debajo de otras disciplinas menos corporales y más intelectuales, o transitar del deber ser exclusivamente masculino a las querellas por la formación física femenina. Se recurre a la prensa pedagógica, pues su crecimiento considerable y el papel que tuvo en la difusión de nuevas tendencias sociales marcarían los procesos de progreso y modernidad, lo cual la convierte ahora en una fuente primaria para el análisis de prácticas culturales, sociales y educativas, en especial las referidas a la educación física, desde el tratamiento metodológico de la historia cultural.
\end{abstract}

Palabras clave: educación física; disciplina escolar; prensa pedagógica

This reflection article aims to analyze the cultural practices of the physical education as part of an education of the body from the school and the gender, during the first decade of the Mexican twentieth century through the pedagogical journals. This starts from the consideration, based on the history of Mexican education, that at the beginning of the twentieth century, the physical education lived one of the most important periods throughout its history. Several processes began to develop around it, considered as school discipline and a daily practice under the shelter of the reforms implemented by the administration of Porfirio Diaz, particularly in the decade of 1900-1910. These reforms generated disputes against it by placing it under other less bodily and more intellectual disciplines or crossing the condition of being exclusively masculine to the quarrels for female physical formation. The pedagogical press is used, since its considerable growth and the role that it had in the diffusion of new social tendencies would mark the processes of progress and modernity, which makes it now a primary source for the analysis of cultural, social and educational practices, especially those relating to physical education, from the methodological treatment of cultural history.

Keywords: physical education; school discipline; pedagogical press

1 Una primera versión de este trabajo fue presentada en el xı Congreso Iberoamericano de Historia de la Educación Latinoamericana, México, 2014.

2 Maestra y licenciada en Pedagogía y profesora de asignatura en la Facultad de Filosofía y Letras, Universidad Nacional Autónoma de México, México. Correo electrónico: cycnus85@yahoo.com.mx 
Este artigo de reflexão tem como objetivo analisar as práticas culturais da educação física como parte de uma educação corporal da escola e do gênero durante a primeira década do século xx mexicano, a partir de revistas pedagógicas. Isso começa com a consideração, a partir da história da educação mexicana, de que, no início do século $\mathrm{XX}$, a educação física passou por um dos períodos mais importantes de sua história. Uma série de processos foi criada em torno dela, considerada a disciplina escolar e a prática cotidiana, sob o abrigo das reformas implementadas pelo presidente Porfirio Díaz, particularmente nas décadas de 1900-1910, que geraram disputas contra ele, colocando-a abaixo de outras disciplinas menos corporais e mais intelectuais, ou transição do deve ser exclusivamente masculino para queixas sobre treinamento físico feminino. Utiliza-se a imprensa pedagógica, pois seu crescimento considerável e o papel que exerceu na difusão de novas tendências sociais marcariam os processos de progresso e modernidade, o que agora a torna uma fonte primária para a análise de práticas culturais, sociais e educacionais; principalmente as referentes à educação física, a partir do tratamento metodológico da história cultural.

Palavras-chave: educação física; disciplina escolar; imprensa pedagógica

Fecha de recepción: 15 de febrero de 2019

Fecha de evaluación: 08 de marzo de 2019

Para citar este artículo:

Ramírez Hernández, G. (2019). La educación física en la prensa pedagógica mexicana en los inicios del siglo xx. Un campo en disputa, lo escolar y el género. Lúdica Pedagógica, 29, 81-90. https://doi.org/10.17227/ ludica.num29-11087 


\section{INTRODUCCIÓN}

La educación física como disciplina escolar y práctica social y cultural ha atravesado diversos momentos a lo largo de la historia de México, de los cuales uno de los más interesantes y notorios es el que se vivió a finales del siglo XIX y principios del xx, quizá hasta la época posrevolucionaria, ${ }^{3}$ pues su puesta en práctica entre la sociedad mexicana la llevó a cambios considerables en los que pasó de ser una actividad poco conocida y practicada a una actividad institucionalizada y escolarizada. La ampliación, al menos en lo concerniente a su difusión, alcanzó incluso a la prensa porfiriana, en especial las revistas pedagógicas dirigidas a los actores de la educación en aquella época, por lo que su presencia cambió a un ámbito más cotidiano y popular.

Estas particularidades también nos llevan a considerar la educación física más allá de una actividad centrada en el movimiento, y su configuración se complejiza como parte de lo que podríamos denominar una educación corporal. Es decir, la educación física funcionó como parte de la conformación de subjetividades referidas a lo corporal a través de la cual se transmitieron e interiorizaron esquemas, ideales, valores, pautas de conducta, costumbres y una gran variedad de signos culturales y sociales con el fin de moldear al sujeto porfiriano, por lo que podríamos hablar de una construcción de la subjetividad referida a la educación física como una educación corporal.

En este sentido, el cuerpo se puede entender más allá de una perspectiva anatómica o biológica y adquiere una connotación cultural y social al ocupar un lugar constitutivo en la formación de los sujetos. Al respecto, Clementina Nava (2011) dice:

3 En la historia de México y en lo referente a lo abordado en este trabajo, dos épocas fueron de suma importancia: el periodo en el que gobierna el general Porfirio Díaz y que se conoce como porfiriato, que se ubica entre finales del siglo xIX y principios del siglo $x x, y$ el correspondiente al periodo revolucionario que da comienzo en 1910 con el conflicto bélico y la época que se podría denominar como posrevolucionaria comprendida entre las siguientes dos décadas (1920-1940 ca.); sin embargo, en estudios recientes, algunos historiadores abordan esta última época también como revolucionaria por dar cuenta de una consolidación de políticas, instituciones y prácticas que se fundamentaron en una ideología nacida de la revolución y se vieron condensadas en una serie de proyectos de diversa índole.
El cuerpo es lo que nos sostiene y nos sustenta: no tenemos un cuerpo, sino, somos un cuerpo, y lograr esta reconciliación es una de las cosas más importantes: que el cuerpo deje de ser concebido [...] como un instrumento para vivir la vida, y sea considerado como la vida misma porque es la esencia del propio ser (p. 9).

Bajo esta premisa, el cuerpo se analiza como un principio de individuación y al mismo tiempo permite un lugar en una configuración colectiva: somos cuerpo individualmente y nos apropiamos del mundo que nos rodea a través de las experiencias corporales, lo que permite la formación de un ser social y cultural. Por ello, es necesario estudiar las prácticas corporales como culturales, donde se depositan aspectos tan amplios como los rasgos políticos, los significados de una diferenciación cultural de las cualidades biológicas de los sexos, entre otros, que tocan de manera inmediata la educación física.

Así, dicha educación, vista como práctica cultural, nos permite un acercamiento metodológico desde la mirada de la historia cultural para poder encontrar en ella la pluralidad de discursos y de tensiones que históricamente la han atravesado. Al mismo tiempo, esa mirada que autores como Peter Burke ${ }^{4}$ nos presentan y funciona como base para este trabajo, nos da pautas para conceptualizar este campo desde fuentes de información tan diversas y particulares que han sido poco trabajadas como lo es la prensa pedagógica, las revistas para y por maestros, directivos y alumnos que circularon entre el cotidiano de la sociedad porfiriana de manera más accesible y a bajo costo, de modo diferente a otras formas de difusión cultural y educativa, y permitieron, entre otros aspectos, el fortalecimiento ideológico de la "educación como panacea para formar ciudadanos y alcanzar el progreso y la felicidad del individuo" (Pérez-Rayón, 2001, p. 211). Entre ellas destacan La Enseñanza Primaria

4 Peter Burke, historiador británico, alude a una historia cultural, desde los postulados de Johan Huizinga (1872-1945), como el "retratar patrones de cultura, es decir, describir los pensamientos y los sentimientos característicos de una época y sus expresiones o encarnaciones" (Burke, 2006, p. 22). Esta definición permite nuevas lecturas e interpretaciones de la concepción histórica del devenir humano más allá de la perspectiva lineal y monumental que ha permeado en la narrativa y la memoria históricas, y da lugar a la pluralidad de la vida social y a las particularidades de los sujetos y sus prácticas como posibles construcciones y acercamientos desde otras miradas metodológicas como lo es esta. 
(1901-1910) y La Enseñanza Normal (1904-1910), las cuales adquirieron una aceptación considerable entre la sociedad, que se puede especular por la prolongada duración de su publicación, y por ser ambos tipos de educación a los que se tomó en cuenta en forma mayoritaria durante el porfiriato y en los que se trabajó más en el ámbito de lo escolar.

A pesar del tránsito que sufrió la prensa entre lo institucional y los medios de difusión, e incluso entre los ámbitos en que los actores educativos se desarrollaban, la tradición histórica de su falta de credibilidad e importancia prevaleció entre el imaginario de la población, y las disputas y tensiones siguieron presentes, no sin dejar cambios sociales tan importantes que aún en la actualidad seguimos practicando, tanto en las instituciones educativas como en los espacios en los que se desarrolla la vida cotidiana de nuestra sociedad mexicana.

Desde esta visión, se presenta este artículo con la intención de dar a conocer, de manera breve, lo que implicó la educación física como un campo de educación corporal desde lo escolar y el género, como parte de una investigación más amplia referida a dicha educación en el porfiriato, ${ }^{5}$ por lo que la inquietud fue analizar las prácticas culturales de la educación física desde la historia referida a la corporalidad y así describir algunas formas que nuestro campo en cuestión atravesó durante la última década del porfiriato, a partir de las revistas pedagógicas como medio de difusión educativa y cultural en la época, y ahora como fuente para la historia de la educación.

\section{ACTIVIDAD Y MOVIMIENTO EN LA ESCUELA}

Durante el porfiriato, la principal aspiración que guio prácticamente todas las esferas sociales fue la del orden y el progreso con vistas a una sociedad moderna, ante todo desde los ojos de las naciones extranjeras, o al menos eso pretendió el General Porfirio Díaz, ${ }^{6}$ quien basó su política gubernamen-

5 La investigación referida resulta mi trabajo de investigación de maestría que derivó en la tesis Educar el cuerpo en el porfiriato (1900-1910 ca.). Una mirada a través de las revistas pedagógicas.

6 Porfirio Díaz Mori (1830-1915) fue presidente de México de 1876 y hasta su exilio a Francia en 1911, con lo cual se convirtió en el mandato más largo en la historia de México, que funcionaría bajo la bandera de orden y progreso en una de las olas modernizadoras que el país ha vivido. tal en mejorar la nación mexicana, modernizarla y hacerla atractiva ante los países más interesantes para él mismo, como lo fue Francia, Alemania, Estados Unidos, entre otros. A lo largo de más de treinta años, las reformas implementadas siguieron la luz del progreso y la modernidad, y la escuela fue uno de los medios principales para lograrlo. A través de ella, se pretendió transmitir los ideales de esta nueva nación que se fortalecieron con la inquietud pedagógica de vigorizar a los individuos a partir de preceptos morales que acompañaban la actividad física, por lo que uno de los grandes logros fue la implementación de la educación física como parte de los planes de estudio de los niveles elementales y de enseñanza normal.

En los albores de lo que conocemos como educación integral, la cultura física tomó un lugar primordial dentro de los preceptos que fundamentaban dicha integralidad, e incluso hablar de educación física podría considerarse como sinónimo, entre algunos especialistas, pues su concepción abarcaba tanto el establecimiento de conductas y hábitos como "las medidas de profilaxis indispensables, por ejercicios corporales apropiados y por la formación de hábitos de higiene" (Anuarios Escolares de la Secretaría de Instrucción Pública y Bellas Artes, 1910-1911, s. p.); es decir, un desarrollo pleno y total del individuo que también podría tener alcances morales, por lo que era evidente la amplitud en su consideración.

A partir de ello, la educación física se concibió como aquella educación que alcanzaba no solo el cuidado, desarrollo y conciencia del cuerpo en movimiento a través de la sistematización de ejercicios físicos, sino también aquella que se encargaba del cuidado de la salud y la higiene, e incluso, en muchas ocasiones, de la transmisión y adquisición de aptitudes y conductas vistas y aceptadas como correctas, aunque siempre estuvo como prioridad el desarrollo físico del individuo, en especial de los infantes; "mediante una educación física sistemática y racional, [se conseguiría] modificar notablemente las condiciones fisiológicas del cerebro, y aumentarlo en capacidad, fuerza y vigor" (La Enseñanza Normal, enero a junio de 1910, p. 351$)^{7}$ para así eliminar vicios y males mentales

7 Para efectos de este trabajo y en razón del uso de una fuente primaria, se darán las referencias de las citas con el nombre de la publicación, los meses con el año y el número de página, pues muchos artículos no cuentan con autor, algunos autores publican en las dos revistas consultadas en el mismo año, y es indispensable dar cuenta del mes de publicación debido a las 
como la neurastenia; todo ello a través de la implementación de la gimnasia y los ejercicios militares como la base de la educación física escolar.

Desde esta concepción, el campo de lo corporal perseguía dos fines primordiales: "proporcionar descanso al cerebro y dar vigor al cuerpo; y los medios de que se [valía eran] ejercicios calisténicos ${ }^{8}$ ó libres ó en aparatos, dados como intermediarios entre las clases" (La Enseñanza Primaria, 1 de julio de 1901, p. 5). Gracias a ello, entonces, se entendía y reafirmaba la práctica de una educación física para fortalecer a la población, mejorar el desarrollo físico de los individuos y vigorizar a una patria deteriorada desde décadas atrás, con el objetivo de hacerla servil a la nueva nación en progreso, pues, como se decía, "en cuerpos débiles y canijos, fisiológicamente depauperados, no se albergan, de ordinario, almas libres, fuertes, seguras de sí mismas y dotadas de enérgicas facultades" (La Enseñanza Normal, julio a diciembre de 1909, p. 356).

Los anteriores fines y objetivos perseguidos por nuestro campo en cuestión se establecieron ante todo en el espacio escolar, por lo que la actualmente conocida asignatura para el desarrollo corporal tuvo sus principios de escolarización hacia finales del siglo , y se instituyeron desde la introducción de la gimnasia y los ejercicios militares como asignaturas tanto en el nivel de la enseñanza primaria como en la enseñanza normal.

La gimnasia, que históricamente tuvo sus inicios en Suecia, ${ }^{9}$ en la última década del porfiriato implicó "formar cuerpos ágiles, fuertes, robustos y sanos,

prohibiciones que la prensa tenía en esa época y a las condiciones de resguardo de los archivos, por lo que a veces, en algunos años, los meses pueden no ser consecutivos. También se conservan la escritura y tratamiento gramatical de los artículos en su estado original como reglas de acentuación o puntuación, pues a través de ellas también se abre la comprensión histórica tanto de la fuente primaria como del contenido.

8 Los ejercicios calisténicos son una forma de ejercicios globales que se desarrollan sin pesas o algún otro aparato, haciendo uso solo del peso corporal y suelen tener la función de calentamiento para la movilidad de las articulaciones.

9 Tuvo sus antecedentes en la gimnasia del maestro sueco Per Henrik Ling quien, al crear el Instituto Real y Central de Gimnasia de Estocolmo, pretendió en él "formular y sistematizar un conjunto de ejercicios gimnásticos con el propósito de hacer de sus conciudadanos, hombres fuertes y robustos, capaces de ingresar al ejército que en aquella época se alistaba á la defensa de la autonomía é integridad de su patria" (La Enseñanza Normal, septiembre de 1908, p. 348), y años más tarde se retomaría en la educación física de nuestro país por la sis- aptos para contener espíritus rectos, sabios y virtuosos" (La Enseñanza Normal, julio a diciembre de 1909, p. 355). Fue la asignatura que entró en los planes escolares con la intención de sistematizar el movimiento a través de ejercicios libres, con o sin aparatos, y que abarcaba tanto la actividad, como el cuidado de la salud; un ejemplo claro es el caso de la gimnasia médica que formaba parte de las tres formas de gimnasia en conjunto con la gimnasia educativa y la gimnasia militar. Así, la gimnasia comienza a ser "considerada como el movimiento que se imprime a todos los miembros del cuerpo en comunicación con sus funciones" (Chávez y López, s. f., p. 9), lo que ahora podríamos definir como educación física en nuestras actividades escolares.

De igual manera, se implementaron los ejercicios militares, lo cual era bastante comprensible si consideramos las diversas disputas bélicas acaecidas a lo largo del siglo xIX con Estados Unidos (1846-1848) y Francia (1838-1839, 1862), así como la formación militar de Díaz, por lo que la militarización alcanzó el espacio escolar a través de este tipo de ejercicios. Con ellos, "había un modelo de escolar que se quería atacar. Era el de los niños con cuerpos débiles, faltos de peso y estatura que los hacía casi inútiles para el trabajo y para defender a la patria en un contexto de constantes conflictos armados" (Chávez y López, s. f., p. 9). A través de la disciplina corporal que se transmitía con estos ejercicios, se pretendió formar al buen soldado que se convertiría en el buen ciudadano, por lo que el ideal primordial fue el de "formar para servir a la patria", en especial desarrollado a través de los ejercicios militares practicados de forma exclusiva por varones.

En todo este panorama, queda claro que los recintos escolares fueron el espacio por excelencia para la práctica de la educación física, la cual se consideró el mejor medio para alcanzar esa fortaleza tan necesaria en la niñez mexicana, y de alguna manera se logró, pero los desplazamientos que nuestro campo tuvo, en muchos casos, estuvieron lejos de ser optimistas, y en gran parte de las implementaciones, y también entre los miembros del sector educativo, se notó la desvalorización y el señalamiento que por tradición ha caracterizado el terreno de lo corporal.

tematización de ejercicios sin aparatos y los movimientos naturales del cuerpo que se denominaron gimnasia sueca. 


\section{EN EL ESPACIO ESCOLAR: LO ACEPTADO Y LO RESTRINGIDO}

Dentro de las publicaciones pedagógicas, como reflejo de lo que se vivía en el entorno social, la educación física se tornó en un tema de preocupación para muchos especialistas en educación. El más importante fue Manuel Velázquez Andrade quien trabajó cerca del profesor Gregorio Torres Quintero y a quien, tanto por interés como por designación, se le atribuyó el cargo de profesor de gimnasia, por lo que quedó al frente de la educación física en nuestro país desde 1901 —después de haber realizado algunos viajes al extranjero que derivaron en un cúmulo de cursos en su formación-. Se le nombró Inspector de Educación Física y su labor, tanto en esta época como durante el periodo como secretario de educación de José Vasconcelos (1921-1924) y hasta su muerte en 1952, le permitió trasladar al terreno educativo diversas inquietudes en relación con la formación corporal, y ser, tanto en las revistas como en los demás ámbitos, el principal actor en la educación física.

No obstante, la ardua labor de Velázquez y otros educadores, el campo de la educación física no fue visto con agrado entre algunos pedagogos, profesores $y$, sobre todo, padres de familia. Algunos creían en los beneficios de la educación física bien practicada y organizada, pero sin dejar de lado la importancia notablemente mayor de otras materias más "intelectuales". Un claro ejemplo es Celso Pineda, quien consideraba lo significativo de llevar a cabo una actividad física bien establecida, aunque a través de ciertos lineamientos y limitaciones, con la intención de lograr descanso y recuperación, lo cual sería el principal objetivo de su puesta en marcha en la escuela; aspectos que muchas veces no se tomaron en cuenta al establecer ejercicios violentos y fatigosos, por lo que Pineda nos dice que "los ejercicios físicos (...) deberían dejarse para las últimas horas de la tarde después de clase poco intelectuales como la escritura, el dibujo, etc." (La Enseñanza Primaria, 1 de julio de 1901, p. 8).

Desde esta visión compartida por algunos maestros y encargados educativos, queda clara la inquietud de alcanzar un mejor desarrollo físico e intelectual en los niños a partir de la sistematización de la educación física en el espacio escolar, pero su lugar, como bien lo plantea Pineda, quedaba relegado con respecto a las asignaturas más intelectuales y, en conjunto con las manuales o mecánicas, debían realizarse en menor tiempo y con menor importancia por ser justo eso: materias mecánicas, físicas o de movimiento.

Por otro lado, a pesar de los esfuerzos por proyectarse en todos los ámbitos sociales como se esperaba, la educación física se practicó en mayor medida en el espacio escolar, y las esperanzas de formar parte de los hogares o de otros espacios potencialmente formativos se fueron desvaneciendo al dejar solo el aspecto de la higiene y la salud, quizá por ser considerada la actividad física como inservible o poco importante para la contribución de otros ideales individuales y colectivos. A final de cuentas, lo físico, por tradición, seguiría ocupando un lugar social menor con respecto a otras prácticas culturales, aunque en la actualidad el panorama sea menos desolador.

Las características que tuvo como disciplina escolar fueron diversas, incluso podemos considerar la etapa del porfiriato como el momento en que se sentaron las bases de la escolarización de la educación física. Ya forma parte de los planes de la enseñanza elemental y la enseñanza normal, y se considera una sistematización propia como disciplina escolar en conjunto con muchas otras, de ahí, la importancia de analizar los procesos y las prácticas ocurridos en dicha etapa.

La organización comenzó desde los kindergarten o las escuelas para párvulos, pues fueron los infantes la principal inquietud en formación de individuos al visualizar a futuro la nación anhelada. La educación física comprendió el desarrollo de las facultades físicas básicas del niño, en especial de la muscular, con la ejecución de ejercicios a través de juegos organizados o libres y movimientos rítmicos acompañados de música.

Los ejercicios físicos para niños de 4 a 6 años se organizaron de la siguiente manera:

1. Marchar, correr, saltar y ejecutar varios movimientos rítmicos acompañados con música.

2. Juegos maternales de Froebel.

3. Juegos para la educación de los sentidos.

4. Movimientos imitativos.- (a) Movimientos familiares observados en la casa, en la calle y en la 
escuela. (b) Movimientos de trabajadores, obreros, artesanos y labradores. (c) Movimientos de imitación de los animales, tales como vuelo, salto, trote, carrera, galope, etc.

5. Juegos en círculo- (a) Juegos de imitación de la naturaleza. (b) Juegos de oficio. (c) Juegos de sociedad. (d) Juegos improvisados sugeridos por cuentos, canciones ó acontecimientos que se presenten en la vida del niño.

6. Juegos de destreza.- Juegos de pelota, cuerda, aros, raqueta, boliche, trompos y canicas.

7. Bailes.

8. Juegos libres ó introduciendo muñecas, papalotes y diversos juguetes pertenecientes á los niños. (La Enseñanza Primaria, 15 de marzo de 1908, p. 287)

El tiempo de los ejercicios era de 20 minutos y los juegos debían llevarse a cabo a mitad de las demás clases o en sesiones matinales, lo cual nos deja ver que, a pesar de considerarse la actividad física como parte del desarrollo infantil, el lugar que ocupaba era de complemento, de descanso, un tanto diferente al lugar y preferencia que otras disciplinas escolares, más intelectuales como diría Pineda, tenían en la escuela porfiriana.

El caso de la enseñanza primaria fue similar en importancia, pero diferente en organización. La labor se acentuó de igual manera que las especificaciones. Las materias enfocadas al cuidado corporal fueron, como ya se dijo, la gimnasia y los ejercicios militares.

El tiempo dedicado era de 30 minutos, por lo regular en las tardes. En las Escuelas Primarias Rudimentales de Varones y para Niñas, la gimnasia o los ejercicios gimnásticos comprendían:

Primer año: movimientos libres, marchas rítmicas y juegos gimnásticos; segundo año: el mismo programa del primer año y ejercicios de bastones; tercer año: el programa del segundo año y ejercicios con mazas; cuarto año: el programa del tercer año y ejercicios con palanquetas (La Enseñanza Normal, julio a diciembre de 1909, p. 356).

La distribución de los ejercicios y la organización horaria cambiaban en función de la escuela y el género. Dos ejemplos se encuentran en la Escuela Pri- maria Industrial “José M. Chávez” y la Escuela Industrial "La Corregidora". En la primera, los horarios estaban organizados para el primer año así: Gimnasia y Juego de 4.15 a 4.45 lunes, miércoles y viernes, Ejercicios Militares martes y jueves, y para segundo año Gimnasia lunes de 5 a 5.30 y viernes de 5.30 a 6 , Ejercicios Militares martes de 5.30 a 6 y jueves de 5 a 5.30. En la segunda, para el primer año Gimnasia de 3.40 a 4 los martes y viernes, y para el segundo año Gimnasia de 3.30 a 3.50 martes y viernes (Anuarios Escolares de Secretaría de Instrucción Pública y Bellas Artes, 1910-1911).

De igual manera, se organizó la educación física en la enseñanza normal e incluso se alcanzó la profesionalización en el área de la gimnasia. En el plan de estudios de la Escuela Normal de Profesores de la Ciudad de México se impartía para el primer año Ejercicios Militares dos veces por semana; para el segundo, tercer y cuarto año, Gimnasia dos veces por semana y Ejercicios Militares dos veces por semana, y para el quinto y sexto año, Gimnasia dos veces por semana (La Enseñanza Normal, 8 de mayo de 1905).

Con este panorama, claramente podemos observar los esfuerzos por la escolarización de la educación física que en nuestros días aún prevalece, quizá con otras denominaciones y cambios, pero la base se vislumbra en esta organización porfiriana. También es necesario tomar en cuenta que, a pesar de la labor, las distinciones son claras con respecto a otras disciplinas escolares que eran consideradas con otras cantidades y cargas horarias al impartirlas más días de la semana o con mayor cantidad de tiempo de clase, así como el lugar que ocupaban en el día escolar; por ejemplo, en la escuela normal, como ya se mencionó, la educación física en sus modalidades ocupaba un lugar en la enseñanza un par de veces a la semana, mientras que disciplinas como español o matemáticas tenían un lugar los cinco días que asistían al recinto escolar. Esto nos permite corroborar el mayor valor que se le daba y continúa dándosele a la mente y su capacidad con respecto al cuerpo, cuando lo cierto es que ambos configuran el todo del sujeto, e incluso las representaciones mentales y el trabajo intelectual conllevan, primero, experiencias corporales, aprendizajes dados desde el contacto físico y el movimiento humano. 


\section{¿Y LAS MUJERES?}

Por último, otra característica que distinguió el proceso de la educación física como campo y disciplina escolar fue la perspectiva de género. Desde épocas antiguas, las cualidades físicas definieron el rol que jugaban tanto hombres como mujeres y el estatus social que se les asignaba. Normalmente eran los varones los fuertes, los capaces de realizar las diversas actividades físicas tanto para el trabajo como para el entretenimiento, e incluso en nuestros días las distinciones se alcanzan a percibir y a pesar de la apertura para acceder a otros espacios y prácticas, la concepción de lo que cada uno debe realizar en el terreno de la corporalidad es evidente, por lo que las distinciones de género siempre van en función de las significaciones corporales.

En esta perspectiva, el porfiriato en su última década no fue la excepción en las consideraciones sobre las diferencias de género, incluso estaban bastante acentuadas para las actividades mentales y de formación e instrucción, pero a pesar de ello la educación corporal también se tomó en cuenta como una educación para las mujeres, y más allá de los pronósticos y una actitud todavía conservadora tanto del régimen como de los diversos grupos sociales, a ellas también se les educó físicamente.

En primer lugar, la figura de la mujer como aprendiz física se inicia a través de las revistas debido al acceso a las publicaciones pedagógicas, incluso algunas estaban destinadas a las "mujeres del hogar", por lo que resultó de importancia difundir ciertas prácticas a través de la prensa.

En segundo lugar, y más importante, estuvo el educar corporalmente a las mujeres como procreadoras de una raza en mejora, por lo que formar mujeres fuertes permitiría proporcionar a la nación hombres serviles y vigorosos. Esta intención educativa tuvo su base en la gimnasia racional la cual era un tipo de gimnasia que se organizaba en función de la edad, el género y la condición social, y desde la cual se preparó a las mujeres para fortalecerlas y dar lugar a buenas familias, buenos hijos. Se esperaba que, a partir de una formación física femenina, por ser ella la encargada, los hogares contaran con otros hábitos de higiene, salud, alimenticios y de fortalecimiento corporal para reducir así los índices de enfermedades y mortandad.
En palabras de Velázquez Andrade, la importancia de la educación física de las mujeres radicaba en que:

Llamada la mujer, por la naturaleza de su sexo, á desempeñar en la conservación de la especie la más cruda y debilitante tarea, importa mucho que su educación física ó corpórea reciba el mayor grado de atención y se base en un conjunto de principios biológicos y en un sistema de gimnasia atlética que respondan exactamente á su delicada y frágil constitución; á sus múltiples y complejas funciones fisiológicas, así como que llenen las exigencias de su existencia como ser culto en medio de una sociedad que no ha podido aún libertarse completamente de un cúmulo de prejuicios que pesan enormemente en contra de su educación actual y que la hace á veces igual al hombre, tanto en la preparación de la lucha por la vida, como en la aspiración por el ejercicio de los mismos derechos (La Enseñanza Primaria, 7 de noviembre de 1907, p. 133).

Estas consideraciones llenan de esperanza el terreno de la educación física mexicana desde la pluralidad, pues la mujer es considerada para una sistematización de actividades corporales en las que se les enseñaban ejercicios rítmicos, con o sin aparatos, libres, correr, saltar e incluso algunos deportes como el 'lawn-tennis' o el 'básquet-ball' que no implicaban movimientos bruscos, violentos o demasiado fuertes, ya que aún existía la inquietud de mantener esa imagen de fragilidad, corporalidad grácil y ternura en ellas por tradición e inquietud social, así que las actividades físicas, que incluían también la danza, eran característicamente estéticos, frágiles y con suavidad, por lo que la educación física femenina también persiguió el ideal pedagógico de la moralidad y el conservadurismo social.

Existieron diferencias entre a lo que la mujer podía acceder de modo disciplinar con respecto a los varones. Las distinciones se hicieron notar en las asignaturas escolares, pues no todo era impartido a varones y mujeres con igualdad. La gimnasia estaba destinada a ambos géneros e incluso la profesionalización alcanzaba a las mujeres, pues el magisterio fue una profesión en particular desarrollada por ellas. Los ejercicios militares estaban destinados solo para los varones debido a que el trabajo corporal asociado a dichos ejercicios contemplaba especificaciones propias del desarrollo del cuerpo en los varones, considerándolos físicamente más capaces que las mujeres 
en el sentido del servicio a la patria, por lo que las diferencias, al menos en la educación física como disciplina escolar, se encontraban muy marcadas entre hombres y mujeres. El establecimiento de estas diferencias se debió a "su estado de menor resistencia y debilidad orgánicas" [el cual] provocaba que los ejercicios fueran limitados en duración, fuerza e intensidad" (Chávez, 2010, p. 35).

Todas estas consideraciones tuvieron desaprobación en personas y grupos tanto de élite como de esferas religiosas y conservadoras. Lo cierto es que, aunque algunos discursos no se concretaron en prácticas de larga duración como el trabajo en el hogar, la educación física de las mujeres formó parte de otra de las grandes tensiones que se vivió en este campo. Sin embargo, a pesar de los choques, las discusiones, las aprobaciones y las desaprobaciones, estas prácticas discursivas contribuyeron a un cambio importante en la educación corporal y en la formación femenina, que de alguna manera han establecido la pauta para que en nuestros días podamos acceder a formas de actividad física dadas tanto para varones como para mujeres. Al mismo tiempo, se abrió el campo a nuevas prácticas antes no consideradas o no existentes como el cambio en una indumentaria más adecuada, la práctica de otros deportes, los hábitos en la escuela o aquellos proporcionados en el hogar. En definitiva, la perspectiva de género fue y sigue siendo una característica en tensión en el campo de la educación física y la sociedad en general.

\section{PARA CONCLUIR}

Cierto es que muchas posturas caracterizan la educación física, la más relevante es la de considerarla una asignatura escolar menos importante que el resto de las que forman parte de los planes de estudio, quizá por desconocimiento o también derivado de la tradición de darle prevalencia a lo racional sobre lo natural, o una satanización del cuerpo que venimos arrastrando desde siglos atrás. Sin embargo, durante el porfiriato fue evidente la inquietud de comenzar nuevos caminos en el campo de la educación física y fue a través de la escuela, como principal escenario de formación, que los cambios más consistentes se vislumbraron.

Los caminos tomados desde las políticas de progreso y modernidad para la configuración nacional del siglo XIX y comienzos del siglo $\mathrm{xx}$ fueron decisivos para vivir una nueva perspectiva de formación física que sigue determinando el quehacer actual, no sin antes haber presenciado diversas tensiones, rupturas y continuidades tan características de un campo en configuración como lo es este. Tensiones que aún existen como el saber qué enseñar, a quiénes formar o dónde llevarla a cabo, y que seguirán, en muchos casos, guiando las normas, prácticas, discursos y procesos de la educación física.

Ante este panorama, nos queda seguir descifrando esos caminos desde la historia de la educación, pues resulta necesario considerar la educación física desde la mirada histórica de los procesos actuales, hacia los múltiples espacios y en los diversos momentos y etapas, con el fin de entender cómo ha derivado en un campo de tensión y así continuar esa configuración tan necesaria para traer a la sociedad la conciencia de que los saberes y las disciplinas corporales también son determinantes en la construcción subjetiva y social.

Por último, cabe resaltar los diálogos y debates desde otras fuentes de información y otras aproximaciones metodológicas sobre esas tensiones surgidas en torno a lo escolar y el género, aspectos que son quizá los más cuestionados en cuanto al cuidado físico, y así contribuir y enriquecer la configuración del campo y la tan necesaria amplitud ideológica en la aceptación de la educación física, no solo como disciplina escolar sino como parte del campo educativo, de la indagación histórica y del diario acontecer en nuestra sociedad mexicana y latinoamericana, diálogos que nos permitan transitar a otras historias aún no descubiertas o resueltas en relación con la educación física para seguir interrogándonos sobre ella.

\section{FUENTES PRIMARIAS}

Anuarios Escolares de la Secretaría de Instrucción Pública y Bellas Artes. (1910). Educación Primaria 1910-1911, México.

La Enseñanza Primaria. Quincenal Pedagógico. Jefe de redacción Gregorio Torres Quintero, 1 de julio de 1901 a 15 de junio de 1910, México.

La Enseñanza Normal. Publicación periódica de la Dirección General de la Enseñanza Normal en el Distrito Federal, Director Alberto Correa, 15 de septiembre de 1904 a enero a junio de 1910. México, Distrito Federal. 


\section{REFERENCIAS}

Bazant, M. (2006). Historia de la educación durante el porfiriato. México: El Colegio de México.

Burke, P. (2006). ¿Qué es la historia cultural? Barcelona: Paidós.

Chávez, M. (2010). Representaciones del cuerpo y el género en la ejercitación física en México, siglos XIX y xx. Alter. Enfoques críticos, 1, 29-41.

García, C. (2011, 17 de marzo) 2008, año de la educación física y el deporte. Una mirada retrospectiva. http:// sites.google.com/site/educacionfisicaenlinea/
López, 0. y Chávez, M. (s/f). Cuerpo, género y Patria: los inicios de la gimnasia escolar mexicana y los textos canónicos que orientaron su enseñanza. México: El Colegio de San Luis.

Nava, C. (2011). El cuerpo de las mujeres: sometimiento y expropiación. Colección Imagina un mundo sin violencia, 8-11.

Pérez-Rayón, N. (2001). México 1900. Percepciones y valores en la gran prensa capitalina. México: Miguel Ángel Porrúa. 